the previous findings of $\mathrm{R}$. Ludford ${ }^{1}$ on the presence of this cell element in tissue cultured cells.

I am grateful to Miss Honor B. Fell for her courteous answers to inquiries about literature.

Zoology Dept., Trinity College,

J. Brontë Gatenby. Dublin, Oct. 19.

Proc. Roy. Soc., B, vol. 101, 1927.

\section{The Specific Heat of Ferromagnetics and} Ewing's Model of the Magnetic Atom.

Prof. W. Gerlach has recently investigated the connexion between the spontaneous magnetism and the specific heat of a ferromagnetic, and he confirms the close relationship between them, pointed out by Prof. P. Weiss, which seems to imply the existence of an immense intrinsic magnetic field within a magnet. The magnitude of this field, however, is so large that there is a difficulty in accounting for its origin from observed magnetic values.

In a paper I submitted to the Philosophical Magazine of March 1922, an experiment was described which showed that an applied alternating magnetic field brings about a loss of ferromagnetism at a lower temperature than the normal critical temperature, leaving unaltered the temperature at which the specific heat suffers a sudden diminution, a temperature which is the same as the normal critical temperature for magnetism.

This almost certainly rules out the hypothesis that the large intrinsic field required to account for the specific heat changes emanates from the rotating magnetic element in a ferromagnetic substance. Hence the suggestion was made in the paper referred to that there must be two fields in a magnet interacting in some way on each other, one concerned with mag. netic quantities as commonly observed and the other with specific heat changes.

Now Ewing's new model of the atom offers a possible solution of some of the difficulties mentioned above and supplies the double field required. In Ewing's words, " Each atom forms a magnetic system comprising a Weber element capable of turning, but controlled by magnetic forces exerted on it by other parts of the atom which are taken as fixed". Mechanically, the model consists of a central pivoted magnetic needle controlled by bar magnets of equal strength, arranged radially in pairs at equal distances around the central needle with poles of one name all facing outwards. The poles pointing inwards produce an almost neutral control on the moving needle as required by the facts of magnetic induction, although the control magnets themselves may be very strong. To make this model fit the facts of ferromagnetism and abnormal specific heat we have only to suppose that it is as likely that the outwardly pointing poles of any atom will be north as south, and so one half the atoms may be supposed to have one polarity externally and the other half the opposite polarity. Between two atoms of opposite polarity there will be a strong attraction and a strong field, and they will tend to join together, forming molecules and groups of molecules. Such molecules or their component atoms, when subjected to thermal agitation, will acquire translatory movements, and these will inevitably set up atomic or molecular oscillations in virtue of the strong magnetic ties between one and another. The Weber element will be constrained to partake of these vibratory movements, and the substance as a whole will appear to lose magnetism and to gain in specific heat as the atoms or molecules successively take up vibrations with rise of temperature.

$$
\text { No. 3241, VoL. 128] }
$$

The model affords an explanation of the experiment mentioned above in which an alternating field applied to a ferromagnetic lowers its critical temperature, but leaves the temperature of the discontinuity of the specific heat unchanged. Such an alternating field would facilitate the vibrations of the Weber element, but would have no influence on the system of 'fixed" atomic magnets. Rupturing of atomic ties with ac. companying loss of potential energy due to violent thermal agitation would account for the discontinuity of the specific heat.

That this model of the ferromagnetic atom should offer a plausible view of how an immense intrinsic magnetic field may exist in a magnet and yet not be influenced by external magnetic forces, and how the magnetism and specific heat of a ferromagnetic may be linked together, is the more noteworthy when it is remembered that the model was devised for an entirely different purpose, namely, the removal of a difficulty occurring in the early stages of magnetic induction with which the older and simpler model was confronted and was incompetent to meet.

Rochdale, Nov. 3.

\section{J. R. Ashworth.}

\section{Homo sapiens and African Prehistory.}

The confirmation by Dr. Leakey and Mr. Hopwood of the antiquity of Hans Reck's Oldaway man, and Mr. Reid Moir's letter in NatuRE of Nov. 7, encourage me to publish, for the first time, a disconcerting discovery made in Uganda about a year ago.

At Nsongegi, in Ankole, the Kagera river is flanked by some remarkably fine terraces of which there are three. The upper and oldest is represented by mere remnants and carries in its upper parts an industry which, though not yet fully studied, is clearly seen to display marked Clactonian affinities, and is dated as Pluvial I. (Leakey's Kamasian). The second, or the hundred foot terrace, like the first, marks a time when in consequence of high precipitation the lakes rose and flooded back into their feeder valleys, and is dated as Pluvial II. (Leakey's Gamblian). Pluvial II. had, it would appear, two rainfall peaks, and during the relatively short period between them lake levels subsided to some extent, and thereby alluvial deposits in the valleys were exposed and became fertile plains, the hunting ground of prehistoric man. This interregnum was sufficiently long to permit of a moderate amount of denudation, and for the freshly exposed land surface to become more or less lateritised before it was again covered by gravels and silts. The temporary land surface yields stone tools, cores, and flakes in amazing abundance, representative of what in Europe would be regarded as two cultures: one of very late Acheulean and the other of early Mousterian facies, and it may be noted in passing that cleavers and some peculiar cores are of common occurrence. It would seem that a somewhat similar culture (the Nanyukian) of unproved but supposedly Interpluvial I. date (that is, between the Kamasian and the Gamblian) has been discovered in Kenya.

A number of pits were put down under my direction through the second terrace just referred to, and in one of them there was discovered in situ, 16 feet below the old occupation site that yields so many artefacts, a 'digging-stone', the usual large, round, flattish stone with a hole pierced through the middle. It was made from a comparatively soft rock (phyllite). Others have, I understand, been found resting on bed rock some 6 feet from the surface on the neighbouring hillsides. The specimen in question was found in wellbedded and quite undisturbed deposits belonging (unless our interpretation is radically wrong) to the 